\title{
The role of anionic amino acids in hydrolysis of poly- $\beta-(1,6)-N$-acetylglucosamine exopolysaccharides by the biofilm dispersing glycosidase Dispersin B
}

\author{
Alexandra P. Breslawec, Shaochi Wang, Crystal Li, and Myles B. Poulin* \\ Department of Chemistry and Biochemistry, University of Maryland, College Park, Maryland 20742, \\ United States \\ *Corresponding Author: Myles B. Poulin \\ E-mail:mpoulin@umd.edu
}

Running title: Anionic amino acids in Dispersin B substrate recognition

Keywords: Biofilm, Glycosidase, Glycoside hydrolase, Carbohydrate Processing, Carbohydrate Chemistry, PNAG/PIA, Biofilm dispersal, Exopolysaccharides, Dispersin B 


\section{Abstract}

The exopolysaccharide poly- $\beta-(1 \rightarrow 6)-N-$ acetylglucosamine (PNAG) is a major structural determinant of bacterial biofilms responsible for persistent and nosocomial infections. The enzymatic dispersal of biofilms by PNAGhydrolyzing glycosidase enzymes, such as Dispersin B (DspB), is a possible approach to treat biofilm dependent bacterial infections. The cationic charge resulting from partial de- $N$-acetylation of native PNAG is critical for PNAG-dependent biofilm formation. We recently demonstrated that DspB has increased catalytic activity with de- $N$ acetylated PNAG oligosaccharides; however, there is still little known about the molecular interaction required for DspB to bind native de- $N$-acetylated PNAG polysaccharides. Here, we analyze the role of anionic amino acids surrounding the catalytic pocket of DspB in PNAG substrate recognition and hydrolysis using a combination of site directed mutagenesis, activity measurements using synthetic PNAG oligosaccharide analogs, and in vitro biofilm dispersal assays. The results of these studies support a model in which bound PNAG is weakly associated with a shallow anionic groove on the DspB protein surface with recognition driven by interactions with the -1 GlcNAc residue in the catalytic pocket. An increased rate of hydrolysis for cationic PNAG was driven, in part, by interaction with D147 on the anionic surface. Moreover, we identified that a DspB mutant with improved hydrolysis of fully acetylated PNAG oligosaccharides correlates with improved in vitro dispersal of PNAG dependent Staphylococcus epidermidis biofilms. These results provide insight into the mechanism of substrate recognition by DspB and suggest a method to improve DspB biofilm dispersal activity by mutation of the amino acids within the anionic binding surface.

\section{Introduction}

In nature, bacteria frequently adopt a sessile lifecycle in response to environmental cues that promote the formation of surface attached biofilms (1). Biofilms consist of bacterial cells embedded in a self-assembled matrix composed of lipids, exported protein, extracellular DNA and exopolysaccharides that are known collectively as the extracellular polymeric substance (EPS) (2). The exact composition of the EPS varies depending on the bacterial species and on environmental factors, but it serves the same function in all contexts: facilitating cell-cell adhesion and acting as a protective barrier (3-6). Bacterial cells within the biofilm are shielded from the host immune response, decontamination and are often resistant to common antibiotic treatments $(5,7-9)$. As a result, biofilms are particularly problematic in hospital settings where biofilm formation contributes to more than half of nosocomial infections (10). Thus, approaches to either prevent biofilm formation or disrupt existing biofilms are being actively pursued to complement traditional antibiotic treatments (11-14).

Exopolysaccharides composed of poly- $\beta-(1 \rightarrow 6)-N$ acetylglucosamine (PNAG) are a major structural constituent of biofilm EPS produced by both Grampositive and Gram-negative human pathogens including Staphylococcus epidermidis $(15,16)$, Staphylococcus aureus (17) Escherichia coli (18), Klebsiella pneumoniae (19) and Acinetobacter baumannii $(20,21)$. PNAG was first identified in clinical isolates of $S$. epidermidis where it is commonly referred to as polysaccharide intercellular adhesin (PIA) due to its role as a primary biofilm adhesin (15). Two major forms of PNAG have been isolated from S. epidermidis. The first is a cationic polysaccharide with approximately $15 \%$ of the $N$-acetylglucosamine (GlcNAc) de- $N$-acetylated, and the second is zwitterionic as a result of both partial de- $N$ acetylation and periodic $O$-succinylation of the GlcNAc residues $(16,22)$. Chemical and enzymatic degradation of PNAG as well as genetic knockouts of key PNAG biosynthetic genes both result in disruption of biofilms and reduced virulence in animal infection models, highlighting the importance of this polysaccharide for biofilm integrity $(8,16,18,19,23-25)$. Glycosidase enzymes that specifically hydrolyze PNAG have the potential to be developed as antibiofilm therapeutics as a result of their ability to disperse biofilms $(11,12,25-27)$.

There have been two PNAG specific glycosidase enzymes identified to date: Dispersin B (DspB) (28) and the glycosyl hydrolase (GH) domain of the bifunctional enzyme PgaB (29). DspB is a native $\beta$ - 
hexosaminidase enzyme of Aggregatibacter actinomycetemcomitans that has been shown to cleave PNAG using both endo- and exoglycosidic cleavage mechanisms depending on the nature of the substrate $(28,30-33)$. PgaB is the bifunctional carbohydrate esterase/glycosyl hydrolase enzyme required for PNAG biosynthesis in Gram-negative bacteria $(24,34)$. The PgaB GH domain catalyzes endoglycosidic cleavage of partially de- $N$ acetylated PNAG substrates containing a glucosamine (GlcN) in the -3 binding site $(29,35)$. Despite interest in these enzymes as biofilm dispersal agents and as treatments for biofilmdependent infections, there is still relatively little known about the specific binding interactions required for recognition of their respective PNAG substrates. This is particularly true for DspB. Efforts to engineer more catalytically active variants of DspB as antibiofilm therapeutics would benefit from detailed information about the specific interactions that contribute to substrate recognition and turnover.

In a recent study, we showed that de- $N$-acetylation of PNAG oligosaccharide analogs influences both the mechanism and rate of hydrolysis of DspB for synthetic substate analogs (ie. 1 and 2) (33). Specifically, we found that trisaccharide $\mathbf{2}$ containing GlcN at the +2 binding site showed a nearly 3-fold faster rate of exoglycosidic cleavage when compared to fully acetylated trisaccharide analog 1. These results indicate that the substrate cationic charge may contribute to substrate recognition by DspB through interactions with anionic amino acids. Here we use a combination of site-directed mutagenesis, enzyme activity assays with synthetic PNAG substrate analogs, and in vitro biofilm dispersal measurements to test this hypothesis and identify amino acids involved in PNAG substrate recognition. These results suggest that mutations outside the DspB catalytic pocket influence PNAG hydrolysis activity and can be used to improve the dispersal of PNAG dependent S. epidermidis biofilms by DspB.

\section{Results}

Cationic charge increases the rate of substrate cleavage by DspB
Our recent studies of DspB activity using synthetic PNAG trisaccharide analogs with defined acetylation patterns (1 and $\mathbf{2}$ ) revealed that specific de- $N$-acetylation patterns influence the rate of substrate hydrolysis by DspB (33). Specifically, hydrolysis of the cationic substrate 2 was nearly 3fold faster than for fully acetylated substrate $\mathbf{1}$. These results suggest a hypothesis that the increased hydrolysis of $\mathbf{2}$ may result from specific charge-charge interaction with the cationic $\mathrm{GlcN}$ in the +2 binding site (Figure 1A). To test this further, a substrate analog 3 containing glucose (Glc) at the +2 site was synthesized. The acetylation pattern of $\mathbf{3}$ is the same as that of analog 2 but lacks the cationic charge at the +2 binding site. Analog 3 was synthesized using a one-pot sequential glycosylation approach developed for the synthesis of 1 and 2 (33), as described in detail in the supporting information.

Reaction progress curves for the hydrolysis of $\mathbf{3}$ by DspB were determined by analyzing the reducingend product distribution by HPLC (33). This assay allows for quantification of the remaining substrate and reducing-end products resulting from hydrolysis of $\mathbf{3}$ based on relative HPLC peak areas using the absorbance of the $S$-tolyl aglycone at 254 $\mathrm{nm}$ (Figure 1B). The reaction progress curves for hydrolysis of $\mathbf{3}$ is consistent with sequential exoglycosidic cleavage of the trisaccharide and is consistent with the mechanism observed previously for hydrolysis of 1 and 2 (33). The observed rate of hydrolysis $\left(k_{\text {obs }}\right)$ was determined by fitting the curve for disappearance of the trisaccharide substrate as a function of time to a single exponential using eq. 1 where $\left[\mathrm{E}_{\mathrm{o}}\right]$ is the initial enzyme concentration, $\left[\mathrm{S}_{\mathrm{o}}\right]$ is the initial trisaccharide concentration and [S] is the trisaccharide concentration remaining at time $t$.

$$
[\mathrm{S}]=\frac{\left[\mathrm{S}_{\mathrm{o}}\right]}{\left[\mathrm{E}_{\mathrm{o}}\right]} \times e^{\left(-k_{\mathrm{obs}} t\right)}
$$

The rate of hydrolysis of $\mathbf{3}$ was nearly identical to that of 1, and 3-fold lower than the rate of hydrolysis of cationic analog 2 (Figure 1C). This supports the hypothesis that the increased hydrolysis rate observed with $\mathbf{2}$ results from recognition of the cationic charge of the substrate, and not simply as a result of deacetylation. To further test this hypothesis and determine the specific interactions responsible for recognition of 
the cationic PNAG analogs, we analyzed the structure of DspB in greater detail.

\section{Negatively charged groove of DspB predicted to bind PNAG}

A crystal structure for the DspB apoprotein was reported in 2005 (36), but structural information regarding substrate binding is lacking. DspB is classified as a Family 20 glycosyl hydrolase according to the Carbohydrate Active enZYmes (CAZy) database (37), and adopts a $(\beta / \alpha)_{8}$ TIM barrel fold, where the predicted catalytic site is found within a pocket $\sim 13 \AA$ deep in the center of the $\beta$-barrel (Figure 2A) $(30,36)$. Previous GH20 enzymes have been shown to use a substrate assisted cleavage mechanism in which the oxygen of the substrate $N$-acetamido group acts as the nucleophile resulting in the formation of an oxazolinium ion intermediate (38). The GlcNAc residue at the site of bond cleavage, the -1 site, adopts an ${ }^{4} \mathrm{E}$ conformation and is contained in a "cage" of conserved aromatic amino acids that serves to orient the $N$-acetamido oxygen for nucleophilic attack $(38,39)$. Two acidic amino acids flank the glycosidic bond, serving as a general acid protonating the leaving group oxygen (E184 in DspB) and as a "base" to stabilize the oxazolinium ion intermediate (D183 in DspB) $(30,40)$. The amino acids in the catalytic site are highly conserved amongst GH20 orthologs, but DspB shares little sequence conservation in the shallow binding surface surrounding the catalytic pocket (Figure 2B) (41). This is not surprising, as the GH20 family contains $\beta$-hexosaminidase enzymes that have activity on a range of substrates, from chito-oligosaccharides (39) to gangliosides (42) and mammalian $N$-glycan $(43,44)$. The only GH20 enzymes with confirmed specificity for the $\beta$ $(1 \rightarrow 6)$-linked GlcNAc of PNAG are the DspB proteins from $A$. actinomycetemcomitans and Actinobacillus pleuropneumoniae $(28,45)$.

Analyzing the electrostatic surface charge of DspB identifies a number of negatively charged amino acids contributing to a shallow anionic groove adjacent to the catalytic pocket (Figure 2C). Three residues in particular, D147, D245, and E248, are located along this anionic groove and are within $\sim 15 \AA$ of the catalytic site, suggesting a possible role in the recognition of cationic PNAG analogs. D147 is located in the loop connecting the $\beta 3$-sheet and $\alpha 3$-helix and is conserved as an anionic amino acid (Asp or Glu) in the GH20 orthologues that are most similar in sequence to DspB. The remaining two residues, D245 and E248, are located within an extension of the $\alpha 6$-helix that is unique to DspB structure and absent in all other GH20 enzymes crystallized to date (supporting information Figure S1).

\section{In silico docking simulations support electrostatic protein-substrate interactions}

Further support for the predicted anionic substrate binding surface was obtained from rigid body docking simulations of DspB binding to methyl glycosides of trisaccharides $\mathbf{1}$ and $\mathbf{2}$ performed using Autodock Vina (46). The highest scoring docked structure for both $\mathbf{1}$ and $\mathbf{2}$ (Figure 2D, E) adopt a nearly identical conformation in which the non-reducing end GlcNAc residue is productively positioned in the catalytic pocket in close proximity to D183 and E184. This predicted binding mode places the 2-NAc or 2- $\mathrm{NH}_{3}{ }^{+}$group of the residue at the +2 site of 1 and $\mathbf{2}$, respectively, within $\sim 3.2 \AA$ of the carboxylate oxygen of D147. This supports the hypothesis that this amino acid recognizes cationic substrates through an electrostatic interaction.

\section{Mutation of anionic amino acids reveals their functional role in substrate recognition}

To evaluate the role of anionic amino acids on PNAG substrate recognition and turnover, we mutated residues D147, D245 and E248 to the corresponding asparagine or glutamine residue or to an alanine. We also analyzed the activity of a D183A catalytic site mutant that has been previously shown to be inactive for hydrolysis of colorimetric PNAG substrate analogs $(30,47)$. The effect of these mutations on DspB specificity was evaluated by analyzing reaction progress curves for the breakdown of synthetic PNAG trisaccharide analogs 1-3 as a function of time. As with our previous studies (33), the reactions were monitored by HPLC using the absorbance at $254 \mathrm{~nm}$ of the $S$ tolyl aglycone to quantify the concentration of remaining substrate and all reducing end products. Obtaining steady state kinetics parameters for the 
DspB mutants was not possible, as the $K_{\mathrm{M}}$ values for all substrates were all $>5 \mathrm{mM}$ and could not be directly determined due to the limited solubility of 1-3 (data not shown). Instead, we directly analyzed reaction progress curves for the enzymatic reaction measured at a single substrate concentration of 1 $\mathrm{mM}$ that is well below $K_{\mathrm{M}}$ for all the mutants. The observed rate $k_{\mathrm{obs}}$ for trisaccharide hydrolysis was determined by fitting the concentration of remaining trisaccharide substrate as a function of time to a single exponential decay using eq. 1, as summarized in Figure $3 \mathrm{~A}-\mathrm{C}$. Under conditions where the $[\mathrm{S}]>>K_{\mathrm{M}}$, the reaction velocity $(v)$ can be accurately described by eq. 2 , where the enzyme specificity constant $k_{\text {cat }} / K_{\mathrm{M}}$ is equal to the observed rate constant $k_{\mathrm{obs}}$, assuming that no substrate or product inhibition is observed (48).

$$
v=\left(\frac{k_{\mathrm{cat}}}{K_{\mathrm{M}}}\right)[\mathrm{S}]
$$

As all of the amino acid substitutions tested in this study, with the exception of D183A, occur outside of the catalytic pocket of DspB, we can assume they will predominantly influence the substrate $K_{\mathrm{M}}$. Thus, for the purpose of this study, it is assumed that the pseudo first-order rate constant $k_{\mathrm{obs}}$ is proportional to substrate binding affinity. As seen in Figure 3A-C, both the D147N and D245N mutants resulted in a slight decrease in catalytic activity $(\leq 30 \%)$ when neutral trisaccharide analogs 1 and 3 were used as substrates. A nearly 3-fold decrease in activity was observed for the D147N mutant with cationic analog 2 , whereas the D245N mutant showed only a small decrease $(\leq 30 \%)$ relative to $\mathrm{DspB}_{\mathrm{wt}}$. This difference can be seen most clearly when we look at the relative reaction rate of the mutants with trisaccharide 2 over the rate with 1 (Figure 3D). Both $\operatorname{DspB}_{\mathrm{wt}}(2.9 \pm 0.1)$ and the D245N mutant $(3.2 \pm 0.1)$ display a $\sim 3$-fold increase in rate with 2 compared to $\mathbf{1}$, whereas the increase is only $1.6 \pm 0.1$ fold for the D147N mutant. This observation is consistent with a role of D147, but not D245, in recognition of cationic PNAG substrates.

The activity of DspB E248Q with cationic analog 2 or Glc containing analog $\mathbf{3}$ were statistically indistinguishable from those of $\mathrm{DspB}_{\mathrm{wt}}$, but showed a 5-fold increase in catalytic activity with fully acetylated trisaccharide analog $\mathbf{1}$. This was rather unexpected, as our hypothesis was that the mutation of anionic amino acids would predominantly affect DspB activity with cationic substrates. Since the enhanced activity was only observed for D248Q with fully acetylated trisaccharide $\mathbf{1}$, it may result from additional hydrogen bonding between the substrate $\mathrm{N}$-acetamido group and the amide side chain of E248Q. To test this hypothesis further, an E248A mutant was prepared. Both the activity and specificity of DspB E248A were indistinguishable from those of $\mathrm{DspB}_{\mathrm{wt}}$ with all substrates tested (Figure 3A-C). This data supports our hypothesis that interactions with the amide side chain of E248Q contributes to the enhanced activity observed with analog 1 .

\section{Role of anionic residues in biofilm dispersal}

The substrate specificity measurements reported in Figure 3 were obtained using synthetic PNAG substrate analogs and may not accurately represent the role of these anionic amino acids in the recognition of native PNAG polysaccharides. To test this, we evaluated the D147N, D245N, D248A, and D248Q mutants for their ability to hydrolyze PNAG in an in vitro model of $S$. epidermidis biofilm dispersal. S. epidermidis RP62A is a methicillin resistant isolate that produces robust PNAG-dependent biofilms on abiotic surfaces (16, $22,49,50)$. Here, biofilms of $S$. epidermidis RP62A that were grown in static culture for 24 hours in a 96-well microtiter plate were treated with increasing concentrations of each DspB mutant and the remaining adherent biofilm biomass was quantified by crystal violet staining (Figure 4A) $(51,52)$. After treating the biofilms for 90 min with $\mathrm{DspB}_{\mathrm{wt}}$, there was a quantifiable reduction in adherent biofilm biomass that was dependent on enzyme concentration. Under these conditions, a biofilm dispersal $\mathrm{EC}_{50}$ of $240 \pm 50 \mathrm{pM}$ was measured for $\mathrm{DspB}_{\mathrm{wt}}$ (Figure 4B). Treating with the catalytically inactive D183A mutant resulted in less than 20\% dispersal, even after 90 min treatment with $2.5 \mu \mathrm{M}$ enzyme, which is consistent with this residue's role in stabilizing formation of the oxazolinium ion intermediate during PNAG hydrolysis (30). As seen in Figure 4B, the $\mathrm{EC}_{50}$ for the D147N mutant $(480 \pm 100 \mathrm{pM})$ was nearly 2fold greater than that of $\mathrm{DspB}_{\mathrm{wt}}$ while those for $\mathrm{D} 245 \mathrm{~N}(320 \pm 85 \mathrm{pM})$ and E248A $(280 \pm 110 \mathrm{pM})$ 
were the same as $\mathrm{DspB}_{\mathrm{wt}}$, within experimental error. This is consistent with the observed activities for these mutants with trisaccharide analogs $\mathbf{1}-\mathbf{3}$. The E248Q mutant had the lowest $\mathrm{EC}_{50}$ at $13 \pm 4 \mathrm{pM}$, consistent with the enhanced catalytic activity observed for this mutant with fully acetylated PNAG analog 1.

\section{Discussion}

Given the importance of PNAG for biofilm integrity and its widespread distribution amongst Gram-positive and Gram-negative bacteria, enzymes that catalyze the hydrolysis of PNAG are potentially useful for treating diverse biofilm infections $(11,12)$. Two PNAG glycosidases, DspB and the $C$-terminal domain of $\mathrm{PgaB}$, have been described to date, but both suffer from relatively low catalytic activity $(29,30,35)$. For example, the activity of DspB measured with a variety of substrate analogs has resulted in observed rates in the order of 2-60 $\mathrm{M}^{-1} \mathrm{~s}^{-1}(30,32)$ nearly $4-6$ orders of magnitude slower than comparable rates observed with other GH20 enzymes (40,53). The poor catalytic activity of DspB likely results from the low binding affinity between DspB and its PNAG substrate. In fact, measurements of DspB binding affinity for PNAG oligosaccharides of varying lengths resulted in measured dissociation constants $\left(K_{\mathrm{d}}\right)$ of between 1-10 $\mathrm{mM}(54)$. Efforts to improve DspB catalytic efficiency would benefit from a detailed analysis of substrate binding.

Our previous studies showed that DspB has nearly 3-fold greater catalytic activity with substrate analog 2 containing GlcN in the predicted +2 binding site compared to a fully acetylated analog 1 (33). In the studies described here, we confirmed this substrate preference and demonstrated that it is the cationic charge of GlcN at the +2 site that is responsible for the increased catalytic activity. This suggests that anionic amino acid residues of DspB contribute to PNAG substrate binding. The results support PNAG binding along a shallow anionic groove on the surface of DspB with D147 contributing to the recognition of cationic GlcN residues in the PNAG substrate. A relatively modest decrease in activity was observed for a D147N mutant, consistent with its role in recognition of cationic PNAG substrates, but also indicating that other residues along the substrate binding surface likely contribute to the recognition of cationic PNAG substrates as well. The reduced activity of DspB D147N with synthetic substrate analogs was consistent with observed biofilm dispersal activity of PNAG-dependent $S$. epidermidis biofilms in a surface attached biofilm model.

Neither of the other anionic amino acids, D245 and E248, appear to contribute to the specificity of DspB for trisaccharide analogs 1-3 or to the in vitro dispersal of $S$. epidermidis biofilms, as D245N and E248A mutants had activity that was indistinguishable from DspB $_{w t}$ in these assays. These results are consistent with the bound conformation of trisaccharide $\mathbf{1}$ and $\mathbf{2}$ predicted from rigid body docking simulations (Figure 2D and E). In these models, the non-reducing GlcNAc residue occupies the catalytic pocket in an orientation consistent with the crystal structures of related GH20 enzymes, including Ostrinia furnacalis Hex1 (55), Serratia marcescens chitobiase (39) Bifidobacterium bifidum lactobiase (56), Streptococcus pneumoniae StrH (44), and Streptomyces plicatus $\beta-N$-hexosaminidase (57). Compared to these GH20 enzymes, the surface surrounding the catalytic pocket of DspB is shallow and lacks aromatic amino acid residues that contribute to substrate recognition through $\mathrm{C}-\mathrm{H} / \pi$ interactions that are present in most of the other GH20 enzymes (Figure S1). The docked conformations of both 1 and $\mathbf{2}$ place the 2-NHAc or $-\mathrm{NH}_{3}{ }^{+}$group of the +2 residue within $3.2 \AA$ of the carboxylate oxygen of D147. Such a conformation would allow for either hydrogen bonding, in the case of 1 , or the formation of a charge-charge interaction, in the case of $\mathbf{2}$, consistent with the reduced catalytic activity observed for the D147N mutant in this study.

Significant interactions were observed between amino acids in the catalytic pocket and the substrate -1 GlcNAc residue and between D147 and the +2 residue, but there was a lack of other stabilizing interactions observed between DspB and the +1 and +2 residues of both docked trisaccharides $\mathbf{1}$ and $\mathbf{2}$. The lack of specific binding interactions helps to explain the relatively poor binding affinity that has been observed between DspB and PNAG analogs in vitro (54). Interestingly, the E248Q mutant, located within the $\alpha 6$-helix extension that is unique 
to the structure of DspB, had no impact on activity with cationic substrate analog 2 , but resulted in a 5fold increase in activity with fully acetylated analog 1. The same activity was not observed for the neutral deacetylated analog 3, supporting the hypothesis that the E248Q mutation introduces new interactions unique to the fully acetylated PNAG substrate. The increased activity was not observed with E248A that lacks the side chain amide, supporting a role for hydrogen bonding between the side chain amide of E248Q and the 2-acetamido group at the +2 site of trisaccharide 1 . Such an interaction was not observed in the docked structure of 1 (Figure 2D) that orients the +2 GlcNAc more than $9 \AA$ from E248. It is important to note that these in silico docking simulations were performed using the DspB apo structure (PDB 1YHT) (36). It is possible that substrate binding induces a conformational change in DspB that would place E248Q in closer proximity to the bound substrate. This conformational flexibility of the $\alpha 6$-helix extension is supported, at least in part, from an analysis of B-factors for the DspB apo structure (36). The $\alpha 6$-helix extension and residues in the anionic binding surface have among the highest Bfactors (Figure S2) indicating these residues reside in an area of increased flexibility in the DspB apo structure. Further structural studies of DspB with PNAG substrate analogs are required to analyze this flexibility in greater detail.

Taken together, the results of the studies presented here support a model in which PNAG binds along a shallow anionic groove on the DspB surface, and D147 contributes to the recognition of cationic PNAG substrates through charge-charge interactions. Neutral, fully $N$-acetylated PNAG likely adopts multiple unique bound conformations within the substrate binding site, and this binding is driven by interactions with GlcNAc at the -1 binding site. Flexibility in the PNAG binding site would accommodate substrates with diverse and heterogeneous PNAG modifications, such as percent de- $N$-acetylation and succinylation, that are known to vary depending on the bacterial species and environmental factors $(16,22)$. Moreover, we showed that site-directed mutagenesis of residues lining this anionic binding surface can alter the catalytic turnover of PNAG substrate analogs such as $\mathbf{1 - 3}$, and that mutations that improve the catalytic efficiency with oligosaccharide substrates correlate with improved dispersal of PNAG dependent biofilms in vitro. Future work should focus on mutations of the residues lining the anionic binding surface to engineer DspB mutants that may function as more effective biofilm dispersal agents for treatment of diverse biofilm-dependent infections.

\section{Experimental Procedures}

\section{Protein production}

Recombinant Dispersin B (residues 16-381, $\mathrm{DspB}_{\mathrm{wt}}$ ) from $A$. actinomycetemcomitans was prepared as described previously (33). Plasmids for expression of $\mathrm{DspB}_{\mathrm{D} 183 \mathrm{~A}}$ were prepared as previously described (47). Plasmids for expression of DspB $B_{\mathrm{D} 147 \mathrm{~N}}, \mathrm{DspB}_{\mathrm{D} 245 \mathrm{~N}}, \mathrm{DspB}_{\mathrm{E} 248 \mathrm{Q}}$, and DspB $\mathrm{B}_{\mathrm{E} 248 \mathrm{~A}}$ mutants were prepared by Quikchange site-directed mutagenesis using the primer pairs outlined in Table 1. All mutations were confirmed by singlepass Sanger sequencing and were expressed recombinantly in E. coli BL21(DE3) and purified as described for $\mathrm{DspB}_{\mathrm{wt}}$ (33). Proteins were quantified by UV absorbance at $280 \mathrm{~nm}$ using a calculated molar extinction coefficient of 51,340 $\mathrm{M}^{-1} \mathrm{~cm}^{-1}$. All proteins were purified to $>95 \%$ purity as confirmed via SDS-PAGE (Figure S3), flash frozen, and stored as individual aliquots at $-80^{\circ} \mathrm{C}$ for later use.

\section{Rigid body docking simulations}

Trisaccharide substrate models for the methyl glycosides of $\mathbf{1}$ and $\mathbf{2}$ were prepared with the nonreducing GlcNAc residue adopting a ${ }^{4} \mathrm{E}$ ring conformation observed in crystal structures of related GH20 enzymes with bound substrate (39, $44,55,57)$. Models for the rest of the trisaccharide substrates were prepared using the lowest energy ${ }^{4} \mathrm{C}_{1}$ ring conformers and glycosidic bond conformations generated using glycan builder on the glycam.org webserver (58), and prepared using in Open Babble. Ring conformations were fixed while all other bonds were allowed to freely rotate. Rigid body docking simulations for the binding of 1 or 2 were carried out using Autodock Vina (46). The highest-scoring ligand conformers for both trisaccharides adopted the same conformation with the non-reducing terminal GlcNAc residue bound 
in the catalytic pocket in the same orientation as observed in other GH20 enzymes. These conformers were exported for figure preparation in Pymol (version 2.3.0).

\section{Time course assays for hydrolysis of PNAG analogs 1-3}

Reaction progress curves for hydrolysis of analogs 1-3 by DspB mutants were measured by HPLC using the previously reported approach with a few modifications (33). Briefly, hydrolysis reactions containing $1 \mathrm{mM}$ of trisaccharide (1-3) in $48 \mathrm{mM}$ potassium phosphate, $\mathrm{pH} 6.0$ buffer containing 100 $\mathrm{mM} \mathrm{NaCl}$ were initiated by the addition of an appropriate concentration of $\mathrm{DspB}_{\mathrm{wt}}$ or $\mathrm{DspB}$ mutant enzyme in a final volume of $50 \mu \mathrm{L}$. Individual $5 \mu \mathrm{L}$ aliquots were removed after $0 \mathrm{~min}$, $10 \mathrm{~min}, 30 \mathrm{~min}, 60 \mathrm{~min}, 90 \mathrm{~min}, 180 \mathrm{~min}, 240 \mathrm{~min}$ and $360 \mathrm{~min}$ incubations at $22{ }^{\circ} \mathrm{C}$ and quenched through the addition of $5 \mu \mathrm{L}$ of $100 \mathrm{mM}$ trifluoroacetic acid (TFA). Quenched fractions were centrifuged at $17,000 \times \mathrm{g}$ for 2 min to pellet any insoluble material, diluted to $50 \mu \mathrm{L}$ with MQ water, and analyzed by reversed phase HPLC as described previously (33). The concentration of residual substrate, and all reducing-end products were determined from their relative peak areas based on the absorbance at $254 \mathrm{~nm}$ resulting from the $S$-tolyl aglycone. Reaction rates for trisaccharide hydrolysis were determined by plotting the concentration of residual trisaccharide as a function of time and fitting to a single exponential using eq. 1 where $[\mathrm{S}]$ is the trisaccharide concentration at time $t,\left[\mathrm{~S}_{\mathrm{o}}\right]$ is the initial trisaccharide concentration, and $\left[\mathrm{E}_{\mathrm{o}}\right]$ is the initial enzyme concentration. This gives a normalized pseudo-first order rate constant $k_{\mathrm{obs}}$ in units of $\mathrm{M}^{-1} \mathrm{~s}^{-1}$.

\section{S. epidermidis biofilm dispersal assays}

S. epidermidis RP62A was obtained from ATCC $\left(\right.$ ATCC $^{\circledR} 35984^{\mathrm{TM}}$ ). For biofilm dispersal assays, a starter culture was grown in $25 \mathrm{~mL}$ of tryptic soy broth (TSB) for $24 \mathrm{~h}$ at $37{ }^{\circ} \mathrm{C}$ with shaking. The starter culture was then diluted to an $\mathrm{OD}_{600}$ of 0.01 using sterile TSB and $200 \mu \mathrm{L}$ of the diluted culture was added to each well of a clear, flat-bottom 96well plate (ThermoFisher Nunc ${ }^{\mathrm{TM}} \mathrm{Edge}^{\mathrm{TM}}$ ). MQ water was added to the outer moat according to the manufactures recommendation to limit evaporation and edge effects during growth. The plates were grown in static culture for $24 \mathrm{~h}$ at $37^{\circ} \mathrm{C}$. After $24 \mathrm{~h}$, the culture media and all non-adherent cells were removed by aspiration and $200 \mu \mathrm{L}$ of sterile $50 \mathrm{mM}$ potassium phosphate buffer, $\mathrm{pH} 6.0$ was added to each well. $20 \mu \mathrm{L}$ of a solution containing various dilutions of $\mathrm{DspB}_{\mathrm{wt}}$ or mutant enzyme in $50 \mathrm{mM}$ potassium phosphate buffer, $\mathrm{pH} 6.0$ was added to the wells to a final volume of $220 \mu \mathrm{L}$. A minimum of eight replicates on each plate were incubated with buffer alone and functioned as no dispersal controls ( $0 \%$ dispersal). The plates were incubated with enzyme at $25{ }^{\circ} \mathrm{C}$ for $90 \mathrm{~min}$, at which time the buffer solution was removed by aspiration and the plates were washed gently but thoroughly with DI water to remove any non-adherent biomass. Remaining adherent cells were fixed with $\mathrm{MeOH}$ $(200 \mu \mathrm{L})$ for one hour after which the wells were aspirated and allowed to fully dry. This was followed by staining with $1 \%$ crystal violet ( 200 $\mu \mathrm{L}$ ) for $5 \mathrm{~min}$. The wells were rinsed thoroughly with DI water until the water ran clear. The plates were imaged to document the stained biofilm biomass.

To quantify the adherent biomass, $200 \mu \mathrm{L}$ of $33 \%$ acetic acid was added to each well to release the crystal violet, and $50 \mu \mathrm{L}$ was removed and diluted with an additional $150 \mu \mathrm{L}$ of $33 \%$ acetic acid in a separate 96 well micro-titer plate. The absorbance of crystal violet in each well was measured at 590 $\mathrm{nm}$ using a plate reader. The relative biofilm dispersal was calculated by dividing the absorbance at $590 \mathrm{~nm}$ by the average absorbance of the no dispersal control wells from each plate. The relative biofilm biomass was plotted as a function of enzyme concentration to determine $\mathrm{EC}_{50}$ values for biofilm dispersal. Each enzyme concentration was analyzed in quadruplicate in different positions on the 96-well plate to minimize artifacts from edge effects. Statistical significance of $\mathrm{EC}_{50}$ values relative to $\mathrm{DspB}_{\mathrm{wt}}$ were determined using a oneway ANOVA with Dunnet's multiple comparison as implemented in GraphPad Prism 8 software.

Data availability: All data supporting the findings in this study are available within the article and its supporting information. 


\section{Author Contributions}

A.P.B. and C.L. produced all proteins, and A.P.B. carried out all activity and in vitro biofilm dispersal assays. S.W. synthesized trisaccharide substrates 1-3. A.P.B. and M.B.P. wrote the manuscript. All authors analyzed data and reviewed the final version of the manuscript.

\section{Acknowledgments}

We are grateful to F. Chen and Y. Li for assistant with analytical NMR and mass spectrometry services.

\section{Funding and additional information}

This work was funded in part by startup funds and a Faculty Student Research Award from the Graduate School at University of Maryland College Park.

Conflict of interest: The authors declare that they have no conflicts of interest with the contents of this article. The content is solely the responsibility of the authors and does not necessarily represent the official views of the National Institutes of Health.

\section{References}

1. Davey, M. E., and O'Toole, G. A. (2000) Microbial Biofilms: from Ecology to Molecular Genetics. Microbiol. Mol. Biol. Rev. 64, 847-867

2. Flemming, H.-C., and Wingender, J. (2010) The biofilm matrix. Nat. Rev. Microbiol. 8, 623-633

3. Costerton, J. W., Stewart, P. S., and Greenberg, E. P. (1999) Bacterial Biofilms: A Common Cause of Persistent Infections. Science. 284, 1318-1322

4. Koo, H., and Yamada, K. M. (2016) Dynamic cell-matrix interactions modulate microbial biofilm and tissue 3D microenvironments. Cur. Opin. Cell Biol. 42, 102-112

5. Nadell, C. D., Drescher, K., Wingreen, N. S., and Bassler, B. L. (2015) Extracellular matrix structure governs invasion resistance in bacterial biofilms. ISME J. 9, 1700-1709

6. Hobley, L., Harkins, C., MacPhee, C. E., and Stanley-Wall, N. R. (2015) Giving structure to the biofilm matrix: an overview of individual strategies and emerging common themes. FEMS Microbiol. Rev. 39, 649-669

7. Vuong, C., Voyich, J. M., Fischer, E. R., Braughton, K. R., Whitney, A. R., DeLeo, F. R., and Otto, M. (2004) Polysaccharide intercellular adhesin (PIA) protects Staphylococcus epidermidis against major components of the human innate immune system. Cell Microbiol. 6, 269-275

8. Vuong, C., Kocianova, S., Voyich, J. M., Yao, Y., Fischer, E. R., DeLeo, F. R., and Otto, M. (2004) A Crucial Role for Exopolysaccharide Modification in Bacterial Biofilm Formation, Immune Evasion, and Virulence. J. Biol. Chem. 279, 54881-54886

9. Yan, J., and Bassler, B. L. (2019) Surviving as a Community: Antibiotic Tolerance and Persistence in Bacterial Biofilms. Cell Host Microbe. 26, 15-21

10. Joo, H.-S., and Otto, M. (2012) Molecular Basis of In Vivo Biofilm Formation by Bacterial Pathogens. Chem. Biol. 19, 1503-1513

11. Kaplan, J. B. (2014) Biofilm matrix-degrading enzymes. Methods Mol. Biol. 1147, 203-13

12. Kaplan, J. B. (2010) Biofilm dispersal: mechanisms, clinical implications, and potential therapeutic uses. J. Dent. Res. 89, 205-18

13. Nijland, R., Hall, M. J., and Burgess, J. G. (2010) Dispersal of Biofilms by Secreted, Matrix Degrading, Bacterial DNase. PLoS ONE. 5, e15668

14. Landini, P., Antoniani, D., Burgess, J. G., and Nijland, R. (2010) Molecular mechanisms of compounds affecting bacterial biofilm formation and dispersal. App. Microbiol. Biotechnol. 86, $813-823$ 
15. Heilmann, C., Schweitzer, O., Gerke, C., Vanittanakom, N., Mack, D., and Götz, F. (1996) Molecular basis of intercellular adhesion in the biofilm-forming Staphylococcus epidermidis. Mol. Microbiol. 20, 1083-1091

16. Mack, D., Fischer, W., Krokotsch, A., Leopold, K., Hartmann, R., Egge, H., and Laufs, R. (1996) The intercellular adhesin involved in biofilm accumulation of Staphylococcus epidermidis is a linear $\beta$-1,6-linked glucosaminoglycan: purification and structural analysis. J. Bacteriol. 178, 175183

17. Cramton, S. E., Gerke, C., Schnell, N. F., Nichols, W. W., and Götz, F. (1999) The Intercellular Adhesion (ica) Locus Is Present in Staphylococcus aureus and Is Required for Biofilm Formation. Infect. Immun. 67, 5427-5433

18. Wang, X., Preston, J. F., and Romeo, T. (2004) The pgaABCD Locus of Escherichia coli Promotes the Synthesis of a Polysaccharide Adhesin Required for Biofilm Formation. J. Bacteriol. 186, 2724-2734

19. Chen, K.-M., Chiang, M.-K., Wang, M., Ho, H.-C., Lu, M.-C., and Lai, Y.-C. (2014) The role of pgaC in Klebsiella pneumoniae virulence and biofilm formation. Microb. Pathog. 77, 89-99

20. Choi, A. H. K., Slamti, L., Avci, F. Y., Pier, G. B., and Maira-Litran, T. (2009) The pgaABCD Locus of Acinetobacter baumannii Encodes the Production of Poly- $\beta-1-6-N$-Acetylglucosamine, Which Is Critical for Biofilm Formation. J. Bacteriol. 191, 5953-5963

21. Guo, H., and Xiang, J. (2015) Influence of poly- $\beta-1-6-N$-acetylglucosamine on biofilm formation and drug resistance of Acinetobacter baumannii. Chin. J. Burn. 31, 45-7

22. Sadovskaya, I., Vinogradov, E., Flahaut, S., Kogan, G., and Jabbouri, S. (2005) Extracellular carbohydrate-containing polymers of a model biofilm-producing strain, Staphylococcus epidermidis RP62A. Infect. Immun. 73, 3007-3017

23. Itoh, Y., Wang, X., Hinnebusch, B. J., Preston, J. F., and Romeo, T. (2005) Depolymerization of $\beta-1,6-N$-acetyl-D-glucosamine disrupts the integrity of diverse bacterial biofilms. J. Bacteriol. 187, 382-387

24. Itoh, Y., Rice, J. D., Goller, C., Pannuri, A., Taylor, J., Meisner, J., Beveridge, T. J., Preston, J. F., and Romeo, T. (2008) Roles of pgaABCD genes in synthesis, modification, and export of the Escherichia coli biofilm adhesin poly- $\beta-1,6-N$-acetyl-D-glucosamine. J. Bacteriol. 190, 36703680

25. Kaplan, J. B., Mlynek, K. D., Hettiarachchi, H., Alamneh, Y. A., Biggemann, L., Zurawski, D. V., Black, C. C., Bane, C. E., Kim, R. K., and Granick, M. S. (2018) Extracellular polymeric substance (EPS)-degrading enzymes reduce staphylococcal surface attachment and biocide resistance on pig skin in vivo. PLoS ONE. 13, e0205526

26. Kaplan, J. B., Ragunath, C., Velliyagounder, K., Fine, D. H., and Ramasubbu, N. (2004) Enzymatic Detachment of Staphylococcus epidermidis Biofilms. Antimicrob. Agents Chemother. 48, 2633-2636

27. Donelli, G., Francolini, I., Romoli, D., Guaglianone, E., Piozzi, A., Ragunath, C., and Kaplan, J. B. (2007) Synergistic Activity of Dispersin B and Cefamandole Nafate in Inhibition of Staphylococcal Biofilm Growth on Polyurethanes. Antimicrob. Agents Chemother. 51, 2733-2740

28. Kaplan, J. B., Ragunath, C., Ramasubbu, N., and Fine, D. H. (2003) Detachment of Actinobacillus actinomycetemcomitans Biofilm Cells by an Endogenous $\beta$-Hexosaminidase Activity. $J$. Bacteriol. 185, 4693-4698

29. Little, D. J., Pfoh, R., Mauff, F. L., Bamford, N. C., Notte, C., Baker, P., Guragain, M., Robinson, H., Pier, G. B., Nitz, M., Deora, R., Sheppard, D. C., and Howell, P. L. (2018) PgaB orthologues contain a glycoside hydrolase domain that cleaves deacetylated poly- $\beta-(1,6)-N$-acetylglucosamine and can disrupt bacterial biofilms. PLoS Pathog. 14, e1006998

30. Manuel, S. G. A., Ragunath, C., Sait, H. B. R., Izano, E. A., Kaplan, J. B., and Ramasubbu, N. (2007) Role of active-site residues of dispersin B, a biofilm-releasing $\beta$-hexosaminidase from a periodontal pathogen, in substrate hydrolysis. FEBS J. 274, 5987-5999 
31. Fekete, A., Borbás, A., Gyémánt, G., Kandra, L., Fazekas, E., Ramasubbu, N., and Antus, S. (2011) Synthesis of $\beta$-(1,6)-linked $N$-acetyl-D-glucosamine oligosaccharide substrates and their hydrolysis by Dispersin B. Carbohydr. Res. 346, 1445-1453

32. Fazekas, E., Kandra, L., and Gyémánt, G. (2012) Model for $\beta-1,6-\mathrm{N}$-acetylglucosamine oligomer hydrolysis catalysed by DispersinB, a biofilm degrading enzyme. Carbohydr. Res. 363, 7-13

33. Wang, S., Breslawec, A. P., Alvarez, E., Tyrlik, M., Li, C., and Poulin, M. B. (2019) Differential Recognition of Deacetylated PNAG Oligosaccharides by a Biofilm Degrading Glycosidase. ACS Chem. Biol. 9, 1998-2005

34. Little, D. J., Poloczek, J., Whitney, J. C., Robinson, H., Nitz, M., and Howell, P. L. (2012) The Structure- and Metal-dependent Activity of Escherichia coli PgaB Provides Insight into the Partial De-N-acetylation of Poly- -1,6-N-acetyl-D-glucosamine. J. Biol. Chem. 287, 31126-31137

35. Forman, A., Pfoh, R., Eddenden, A., Howell, P. L., and Nitz, M. (2019) Synthesis of defined monode- $N$-acetylated $\beta$ - $(1 \rightarrow 6)-N$-acetyl-D-glucosamine oligosaccharides to characterize PgaB hydrolase activity. Org. Biomol. Chem. 17, 9456-9466

36. Ramasubbu, N., Thomas, L. M., Ragunath, C., and Kaplan, J. B. (2005) Structural Analysis of Dispersin B, a Biofilm-releasing Glycoside Hydrolase from the Periodontopathogen Actinobacillus actinomycetemcomitans. J. Mol. Biol. 349, 475-486

37. Lombard, V., Ramulu, H. G., Drula, E., Coutinho, P. M., and Henrissat, B. (2013) The carbohydrate-active enzymes database (CAZy) in 2013. Nucleic Acids Res. 42, D490-D495

38. Mark, B. L., Vocadlo, D. J., Knapp, S., Triggs-Raine, B. L., Withers, S. G., and James, M. N. G. (2001) Crystallographic Evidence for Substrate-assisted Catalysis in a Bacterial $\beta$-Hexosaminidase. J. Biol. Chem. 276, 10330-10337

39. Tews, I., Perrakis, A., Oppenheim, A., Dauter, Z., Wilson, K. S., and Vorgias, C. E. (1996) Bacterial chitobiase structure provides insight into catalytic mechanism and the basis of Tay-Sachs disease. Nat. Struct. Biol. 3, 638-648

40. Williams, S. J., Mark, B. L., Vocadlo, D. J., James, M. N. G., and Withers, S. G. (2002) Aspartate 313 in the Streptomyces plicatus Hexosaminidase Plays a Critical Role in Substrate-assisted Catalysis by Orienting the 2-Acetamido Group and Stabilizing the Transition State. J. Biol. Chem. 277, 40055-40065

41. Ashkenazy, H., Abadi, S., Martz, E., Chay, O., Mayrose, I., Pupko, T., and Ben-Tal, N. (2016) ConSurf 2016: an improved methodology to estimate and visualize evolutionary conservation in macromolecules. Nucleic Acids Res. 44, W344-W350

42. Sumida, T., Ishii, R., Yanagisawa, T., Yokoyama, S., and Ito, M. (2009) Molecular Cloning and Crystal Structural Analysis of a Novel $\beta$ - $N$-Acetylhexosaminidase from Paenibacillus sp. TS12 Capable of Degrading Glycosphingolipids. J. Mol. Biol. 392, 87-99

43. Jiang, Y.-L., Yu, W.-L., Zhang, J.-W., Frolet, C., Guilmi, A.-M. D., Zhou, C.-Z., Vernet, T., and Chen, Y. (2011) Structural Basis for the Substrate Specificity of a Novel $\beta-N-$ Acetylhexosaminidase StrH Protein from Streptococcus pneumoniae R6. J. Biol. Chem. 286, 43004-43012

44. Pluvinage, B., Higgins, M. A., Abbott, D. W., Robb, C., Dalia, A. B., Deng, L., Weiser, J. N., Parsons, T. B., Fairbanks, A. J., Vocadlo, D. J., and Boraston, A. B. (2011) Inhibition of the Pneumococcal Virulence Factor StrH and Molecular Insights into $N$-Glycan Recognition and Hydrolysis. Structure. 19, 1603-1614

45. Kaplan, J. B., Velliyagounder, K., Ragunath, C., Rohde, H., Mack, D., Knobloch, J. K.-M., and Ramasubbu, N. (2004) Genes Involved in the Synthesis and Degradation of Matrix Polysaccharide in Actinobacillus actinomycetemcomitans and Actinobacillus pleuropneumoniae Biofilms. $J$. Bacteriol. 186, 8213-8220

46. Trott, O., and Olson, A. J. (2010) AutoDock Vina: improving the speed and accuracy of docking with a new scoring function, efficient optimization, and multithreading. J. Comput. Chem. 31, 45561 
47. Wang, S., Breslawec, A. P., Li, C., and Poulin, M. B. (2020) A Colorimetric Assay to Enable HighThroughput Identification of Biofilm Exopolysaccharide-Hydrolyzing Enzymes. Chem. Eur. J. doi: $10.1002 /$ chem. 202002475

48. Stroberg, W., and Schnell, S. (2016) On the estimation errors of $\mathrm{K}_{\mathrm{M}}$ and $\mathrm{V}$ from time-course experiments using the Michaelis-Menten equation. Biophys. Chem. 219, 17-27

49. Mack, D., Siemssen, N., and Laufs, R. (1992) Parallel induction by glucose of adherence and a polysaccharide antigen specific for plastic-adherent Staphylococcus epidermidis: evidence for functional relation to intercellular adhesion. Infect. Immun. 60, 2048-2057

50. Christensen, G. D., Bisno, A. L., Parisi, J. T., McLaughlin, B., Hester, M. G., and Luther, R. W. (1982) Nosocomial Septicemia Due to Multiply Antibiotic-Resistant Staphylococcus epidermidis. Ann. Intern. Med. 96, 1

51. O'Toole, G. A., and Kolter, R. (1998) Initiation of biofilm formation in Pseudomonas fluorescens WCS365 proceeds via multiple, convergent signalling pathways: a genetic analysis. Mol. Microbiol. 28, 449-461

52. Stepanović, S., Vuković, D., Dakić, I., Savić, B., and Švabić-Vlahović, M. (2000) A modified microtiter-plate test for quantification of staphylococcal biofilm formation. J. Microbiol. Meth. 40, $175-179$

53. Vocadlo, D. J., and Withers, S. G. (2005) Detailed Comparative Analysis of the Catalytic Mechanisms of $\beta$ - $N$-Acetylglucosaminidases from Families 3 and 20 of Glycoside Hydrolases. Biochemistry. 44, 12809-12818

54. Eddenden, A., Kitova, E. N., Klassen, J. S., and Nitz, M. (2020) An Inactive Dispersin B Probe for Monitoring PNAG Production in Biofilm Formation. ACS Chem. Biol. 15, 1204-1211

55. Liu, T., Zhang, H., Liu, F., Wu, Q., Shen, X., and Yang, Q. (2010) Structural Determinants of an Insect $\beta$ - $N$-Acetyl-D-hexosaminidase Specialized as a Chitinolytic Enzyme. J. Biol. Chem. 286, $4049-4058$

56. Ito, T., Katayama, T., Hattie, M., Sakurama, H., Wada, J., Suzuki, R., Ashida, H., Wakagi, T., Yamamoto, K., Stubbs, K. A., and Fushinobu, S. (2013) Crystal Structures of a Glycoside Hydrolase Family 20 Lacto- $N$-biosidase from Bifidobacterium bifidum. J. Biol. Chem. 288, 1179511806

57. Mark, B. L., Wasney, G. A., Salo, T. J. S., Khan, A. R., Cao, Z., Robbins, P. W., James, M. N. G., and Triggs-Raine, B. L. (1998) Structural and Functional Characterization of Streptomyces plicatus $\beta$ - $N$-Acetylhexosaminidase by Comparative Molecular Modeling and Site-directed Mutagenesis. $J$. Biol. Chem. 273, 19618-19624

58. Woods Group. (2005-2020) GLYCAM Web. Complex Carbohydrate Research Center, University of Georgia, Athens, GA. (http://glycam.org)

59. Ren, B., Cai, L., Zhang, L.-R., Yang, Z.-J., and Zhang, L.-H. (2005) Selective deacetylation using iodine-methanol reagent in fully acetylated nucleosides. Tetrahedron Lett. 46, 8083-8086 
Table 1. Primers for site directed mutagenesis used in this study.

\begin{tabular}{cll}
\hline DspB mutant & & Primer pair \\
& Fwd & 5'- GCCGTCAAGTGGACAATGAGATCGACATTACC \\
D147N & Rev & 5'- GGTAATGTCGATCTCATTGTCCACTTGACGGC \\
& Fwd & 5'- CGGTGATACCCAAAATAAAAACGAGGCGGCGG \\
D245N & Rev & 5'- CCGCCGCCTCGTTTTTCTGTTGGGTATCACCG \\
& Fwd & 5'- CGAGGCGGCGCAACGTCGTGATATGCG \\
E248Q & Rev & 5'- CGCATATCACGACGTTGCGCCGCCTCG \\
& Fwd & 5'- CGAGGCGGCGGACGTCGTGATATGCG \\
E248A & Rev & $5 '-$ CGCATATCACGACGTGCCGCCGCCTCG \\
\hline
\end{tabular}




\section{Figures}
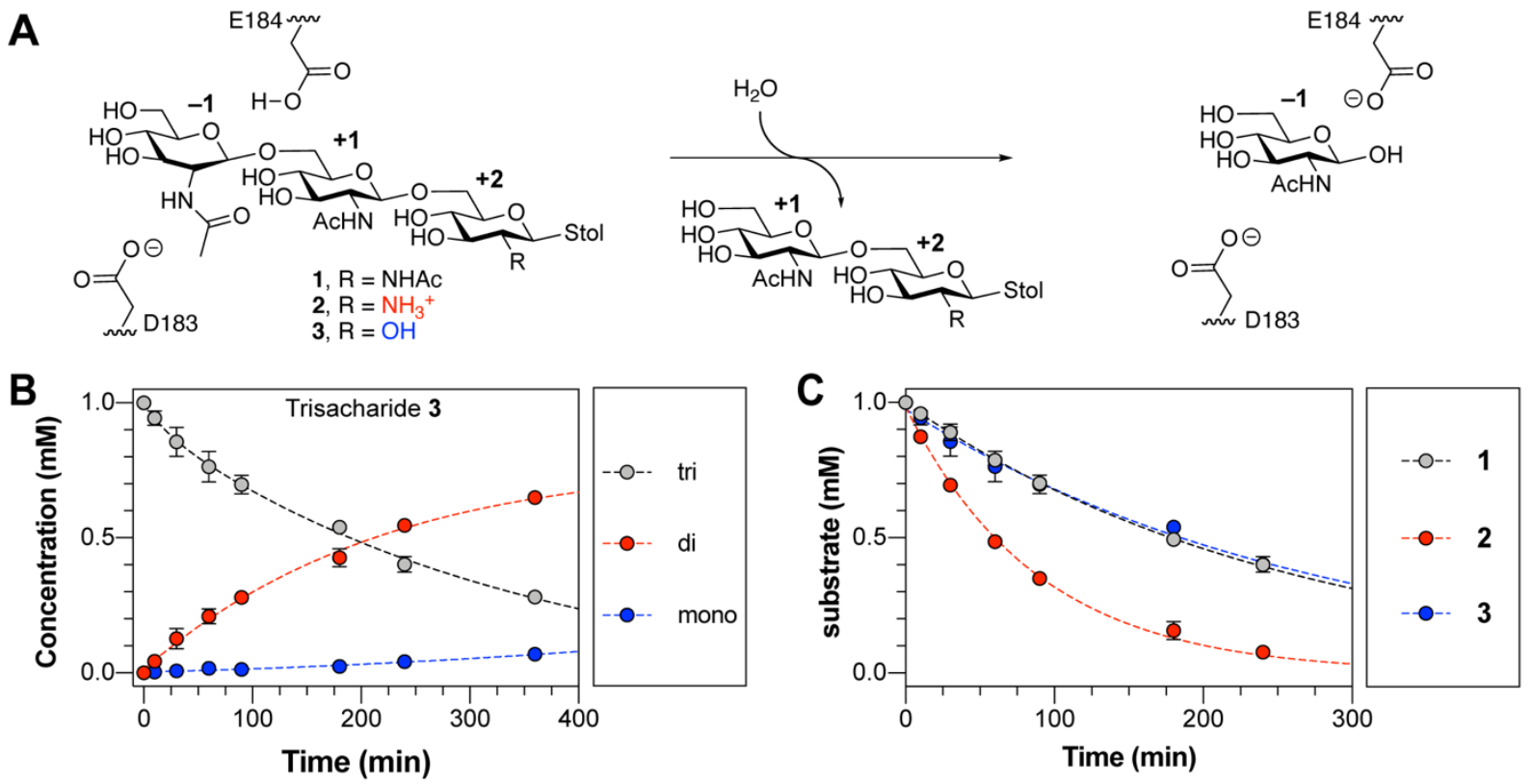

Figure 1. Hydrolysis of PNAG analogs by DspB. A. Reaction catalyzed by DspB showing the major exoglycosidic cleavage activity for trisaccharide analogs 1-3 used in this study. The monosaccharide residues are numbered relative to the site of glycosidic bond cleavage. The position of E184, which serves as a general acid to protonate the leaving group oxygen, and D183, which acts as a base to stabilize the oxazolinium ion intermediate, are shown. B. Reaction progress curve for the hydrolysis of trisaccharide 3 by DspB. Lines were added to aid identification of the disappearance of the trisaccharide (grey) and appearance of reducing-end disaccharide (red) and reducing-end monosaccharide (blue) products from sequential exoglycosidic cleavage of $\mathbf{3}$. Error bars represent the standard deviation from at least two replicate experiments. C. Relative rates of trisaccharide disappearance for hydrolysis of analogs 1-3 by DspB. The rate of trisaccharide disappearance was fit to a single exponential using eq. 1. Error bars represent the standard deviation from two replicate experiments. 
A

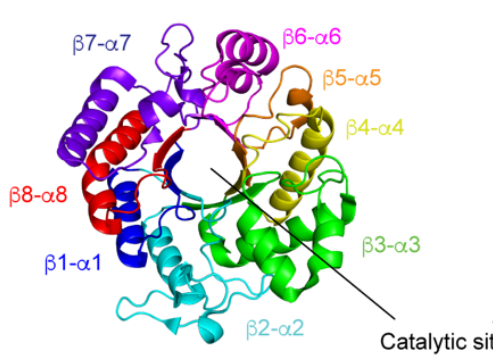

C

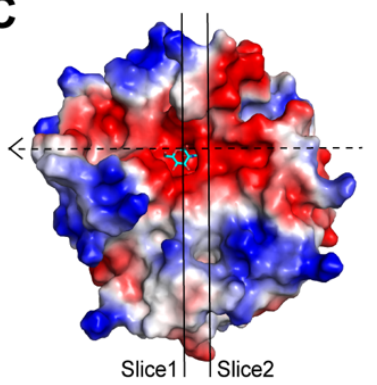

B

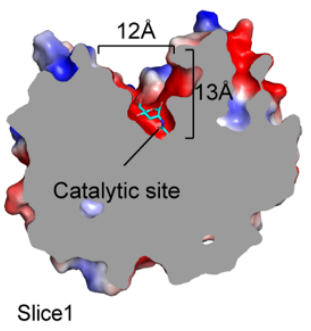

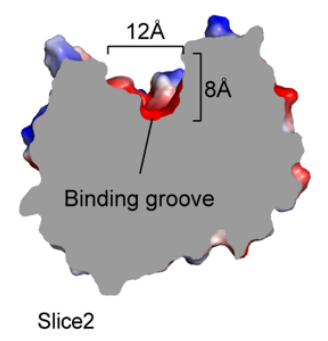

D

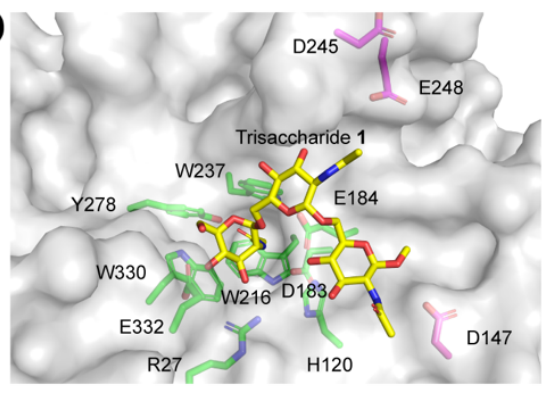

E

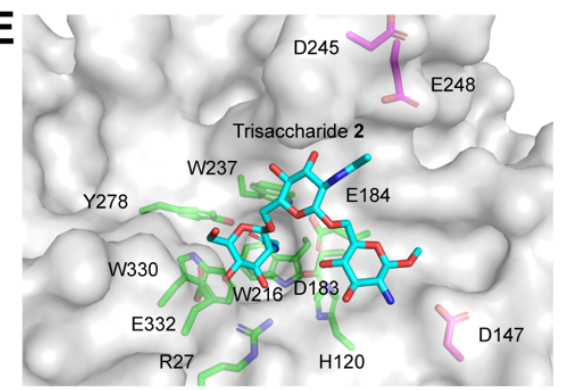

Figure 2. Analysis of the DspB substrate binding surface. A. Structure of DspB (1HYT) showing the $(\beta / \alpha)_{8}$ TIM barrel fold with each $\beta$-sheet and $\alpha$-helix pair highlighted. The position of the putative catalytic site is indicated. B. Surface representation of DspB structure where the residues are colored based on sequence conservation amongst GH20 orthologs as calculated by Consurf (41). Cyan, white and fuchsia indicate regions of low, medium and high sequence conservation, respectively. The position of GlcNAc in the -1 site is shown in cyan sticks. C. Electrostatic surface map of DspB highlighting a shallow $12 \AA$ wide anionic groove on the protein surface (indicated by dashed arrow). Two slices are shown that highlight the approximate dimensions of the binding groove. D-E. Low energy conformations of the methyl aglycones of trisaccharide 1 (D) and trisaccharide 2 (E) bound to DspB. 

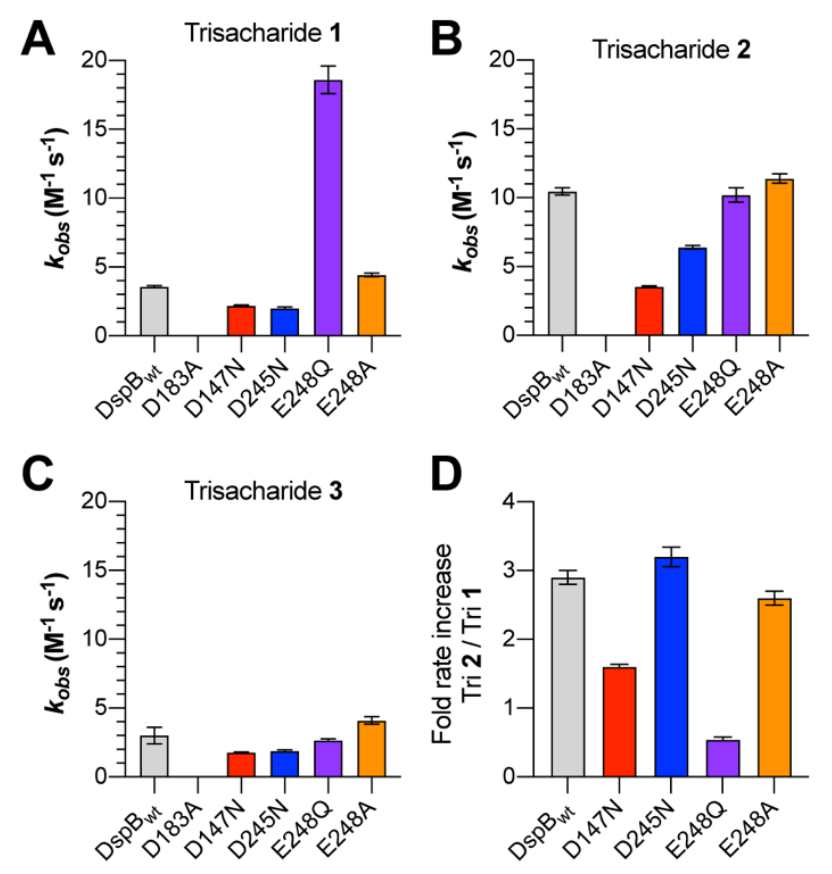

Figure 3. Observed rate of PNAG analog hydrolysis by DspB mutants. A-C. Observed rate constants $\left(k_{\mathrm{obs}}\right)$ for the hydrolysis of trisaccharide 1 (A), 2 (B) and $\mathbf{3}$ (C) by DspB mutants determined by fitting the reaction progress curves for the disappearance of trisaccharide substrate to eq. 1. Error bars represent the standard deviation from two replicate time course measurements. D. Plot of the relative rate with cationic trisaccharide $\mathbf{2}$ compared to fully acetylated trisaccharide $\mathbf{1}$. 

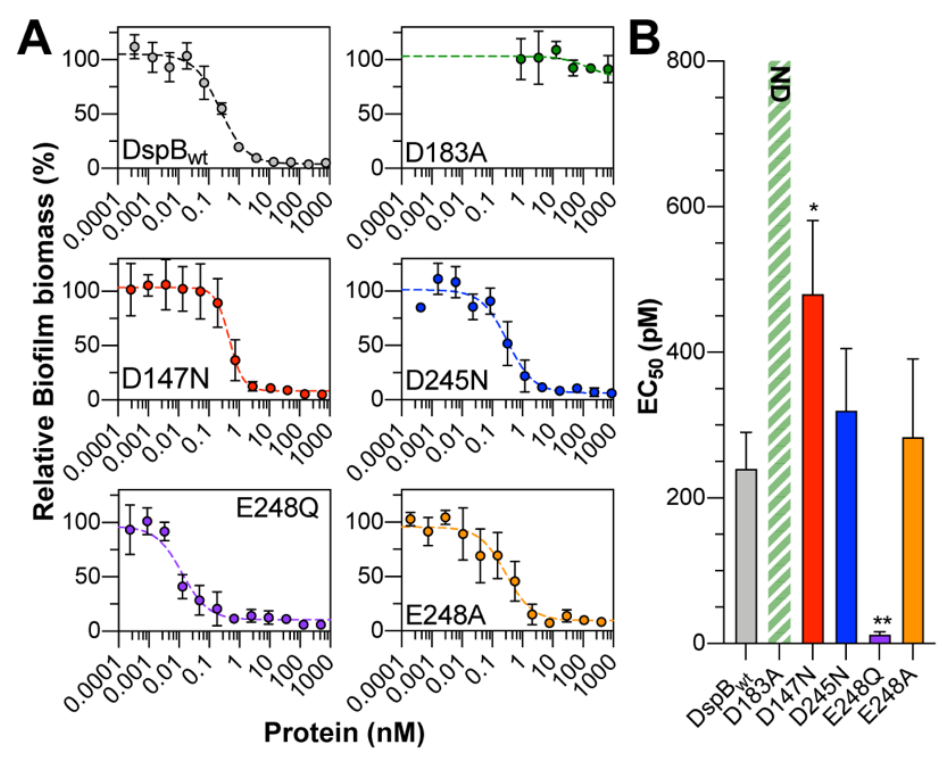

Figure 4. Dispersal of S. epidermidis biofilms by DspB mutants. A. Plots of S. epidermidis RP62A biofilm biomass remaining after treating with varying concentrations of DspB mutants for 90 min relative to untreated biofilms. Error bars represent the standard deviation of four biological replicates. B. EC Ev values $_{5}$ from $S$. epidermidis biofilm dispersal assays. Error bars represent the standard deviation from four biological replicates. Statistical significance as compared to $\mathrm{DspB}_{\mathrm{wt}}$ was determined using a one-way ANOVA with Dunnett's multiple comparison. *, $\mathrm{P}<0.05 ; * *, \mathrm{P}<0.01$. ND: the $\mathrm{EC}_{50}$ was not determined as $>75 \%$ biofilm biomass remained even after treating with $2.5 \mu \mathrm{M}$ enzyme for $90 \mathrm{~min}$. 